\title{
UV-photoactivation technique for size and shape controlled synthesis and annealing of stable gold nanoparticles in micelle
}

\author{
MADHURI MANDAL, SUBRATA KUNDU, SUJIT KUMAR GHOSH and \\ TARASANKAR PAL* \\ Department of Chemistry, Indian Institute of Technology, Kharagpur 721 302, India
}

\begin{abstract}
Gold nanoparticles of different sizes and shapes have been prepared by UV-photoactivation technique using the micelle TX-100 (poly(oxyethylene) iso-octylphenyl ether) as reducing agent, stabilizing agent as well as template which has been authenticated from the plasmon absorption band and TEM picture. The heating effect on those gold nanoparticles has also been studied.
\end{abstract}

Keywords. TX-100; gold nanoparticles; annealing.

\section{Introduction}

Nanoparticle research has become the subject of novel interest in science and technology because these particles of 'neglected dimension' exhibit special properties in many aspects compared to their bulk e.g. catalysis (Pradhan et al 2001), size and shape dependent optical properties (Jana et al 2001), electronic properties (Link and El-Sayed 1999; Butte et al 1999), etc. Metallic nanoparticles, especially the coinage metals, have mainly been studied because of their strong optical absorption in the visible region and they have shown promise in catalysis (Pradhan et al 2001) and SERS studies (Pal et al 2000). Many theories have been put forward to explain the observed experimental behaviour (Kreibig and Vollmer 1995) yet the quantitative answer still remains to be written.

In this article we have addressed successfully the use of micelle as template/capping agent as it has an advantage due to its stabilizing property as well as the growth controlling capacity for the aggregation of particles (Esumi et al 1995). Of all the methodologies developed for the preparation of metal nanoparticles on either physical or chemical ways, the photoactivation technique offers a very simple way by which almost monodispersed (tight size distribution) metal nanoparticles can be formed (Pal 1998). We have successfully applied this technique, for the first time to prepare gold nanoparticles of different shapes and sizes in TX-100 micelle by varying its concentration. TX-100, here, simultaneously acts as a reducing agent as well as stabilizer and template also.

\footnotetext{
*Author for correspondence
}

\section{Experimental}

\subsection{Reagents and instruments}

All the reagents used were of AR grade. Chloroauric acid was purchased from Johnson Matthey, Royston, Hestfordshire, UK. All the solutions were prepared in double distilled water and diluted to different concentrations as necessary. All the absorbance measurements were carried out in Shimadzu UV-160 digital spectrophotometer taking the solution in $1 \mathrm{~cm}$ well stoppered quartz cuvette. Photoirradiations were carried out in a photoreactor fitted with ordinary germicidal lamps (Sankyo, Denki, Japan) of UVC G8 T5.

\subsection{Preparation}

An aliquot of standard $\mathrm{HAuCl}_{4}$ solution and TX-100 solution were mixed together with varying concentrations of TX-100 solution as well as $\mathrm{Au}(\mathrm{III})$ ion solution. Five sets of mixtures were prepared. In set I, the final concentration of $\mathrm{Au}$ (III) ion solution was $0.95 \times 10^{-3} \mathrm{~mol} \mathrm{dm}^{-3}$ and concentration of TX-100 was $0.81 \times 10^{-1} \mathrm{~mol} \mathrm{dm}^{-3}$. In other four sets (II, III, IV, V) the concentration of $\mathrm{Au}(\mathrm{III})$ ion solutions were same, $3.9 \times 10^{-5} \mathrm{~mol} \mathrm{dm}^{-3}$, and the concentration of TX-100 solution was varied. The concentration of TX-100 was adjusted to $0.99 \times$ $10^{-1} \mathrm{~mol} \mathrm{dm}^{-3}, 0.99 \times 10^{-2} \mathrm{~mol} \mathrm{dm}^{-3}, 1.6 \times 10^{-4} \mathrm{~mol} \mathrm{dm}^{-3}$ and $0.79 \times 10^{-5} \mathrm{~mol} \mathrm{dm}^{-3}$ for sets II, III, IV and V, respectively. All the solutions were taken separately in $1 \mathrm{~cm}$ quartz cuvette and irradiated for $25 \mathrm{~min}$ (under UV light of wavelength $\sim 365 \mathrm{~nm}$ ) in the photoreactor. The heating effect on those metal nanoparticles were studied by heating the particles at $\sim 60^{\circ} \mathrm{C}$ in a water bath. 


\section{Results and discussion}

The micelle having an alcoholic skeleton homogeneously reduces $\mathrm{Au}(\mathrm{III})$ ions without increasing the local concentration of the added reagents (Gachard 1998). Thus the photoirradiation inherits the elegant reproducibility with the homogeneous flux density of the light source unlike the wet chemical technique.

Here we have been able to show how varying the concentration of the surfactant can be used as template/ capping agent for the preparation of not only of different size, different shaped gold nanoparticles also. The size of the particles can be easily altered just by changing the concentration of TX-100 solution. For the sets II, III, IV, $\mathrm{V}$ we obtained the particles of average sizes $3 \mathrm{~nm}$, $4.5 \mathrm{~nm}, 7 \mathrm{~nm}$ and $12 \mathrm{~nm}$, respectively. In figure 1 , the

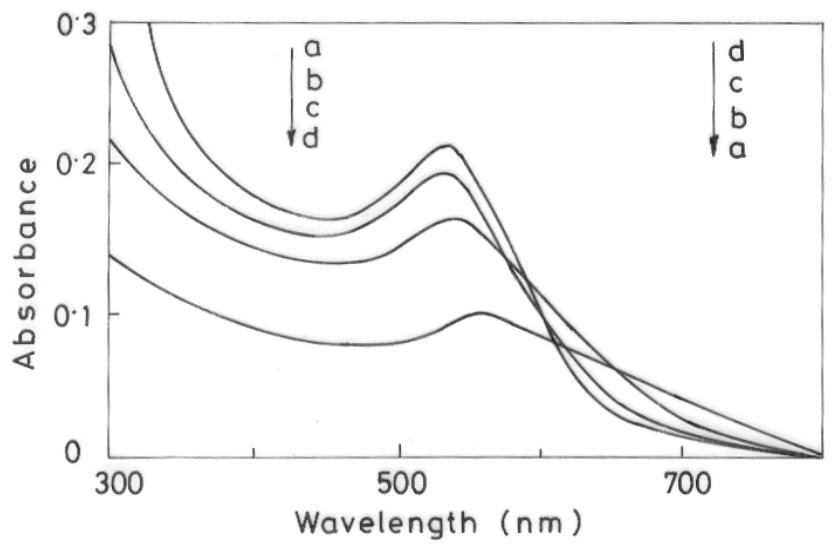

Figure 1. Plasmon absorption band for sets II and V. (Conditions: For all sets $\left[\mathrm{HAuCl}_{4}\right]=3.9 \times 10^{-5} \mathrm{~mol} \mathrm{dm}^{-3}$ and concentration of TX-100 was adjusted to $0.99 \times 10^{-1} \mathrm{~mol} \mathrm{dm}^{-3}$ and $0.79 \times 10^{-5} \mathrm{~mol} \mathrm{dm}^{-3}$ for sets II and $\mathrm{V}$, respectively).
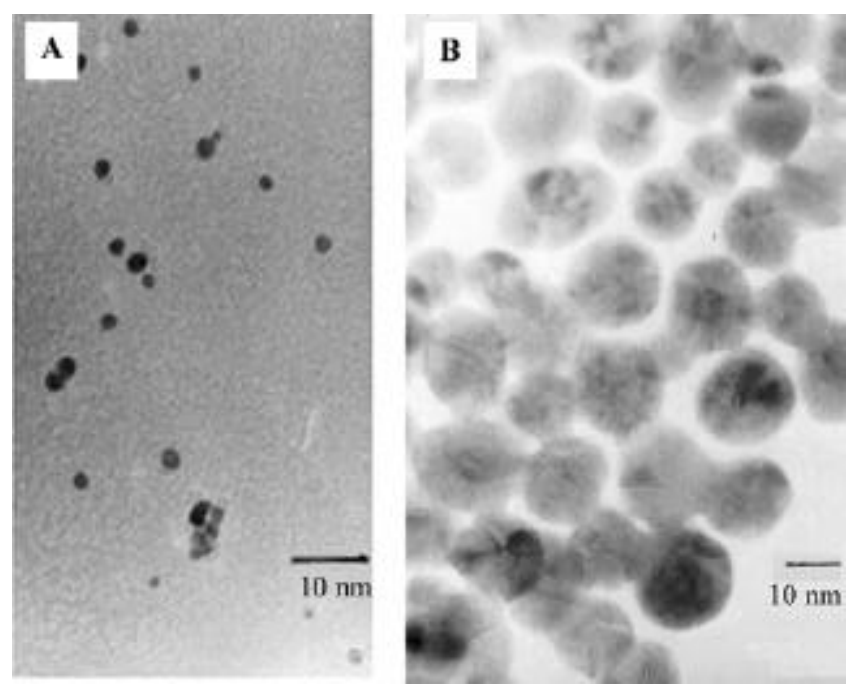

Figure 2. TEM pictures $\mathbf{A}$ and $\mathbf{B}$ for gold nanoparticles for sets II and V, respectively. plasmon absorption band for $\mathrm{Au}(0)$ sol prepared in different micellar concentration has been shown. From this we observed that with decrease in concentration of TX$100, \lambda_{\max }$ shifts to a red region, bandwidth become wider and absorbance value decreased. From this characteristic plasmon band we can conclude that particle size decreases with increase of TX-100 concentration, which was authenticated from TEM measurement (figures $2 \mathrm{~A}$ and $\mathrm{B})$. Keeping the $\mathrm{Au}(\mathrm{III})$ ion concentration unaltered one can have particle of larger dimension for gold particles with the lowering of surfactant concentration. This clearly tells us that the particle growth is spontaneous under less restricted or less hindered environment i.e. in less concentrated surfactant media. Taking higher concentrations of the TX-100 and $\mathrm{HAuCl}_{4}$ solution in comparison to other sets we obtained gold particles of different shapes like rod, cubical, tetragonal along with spherical particles. This is due to the fact that the successive growth of gold nanoparticles has a natural tendency to

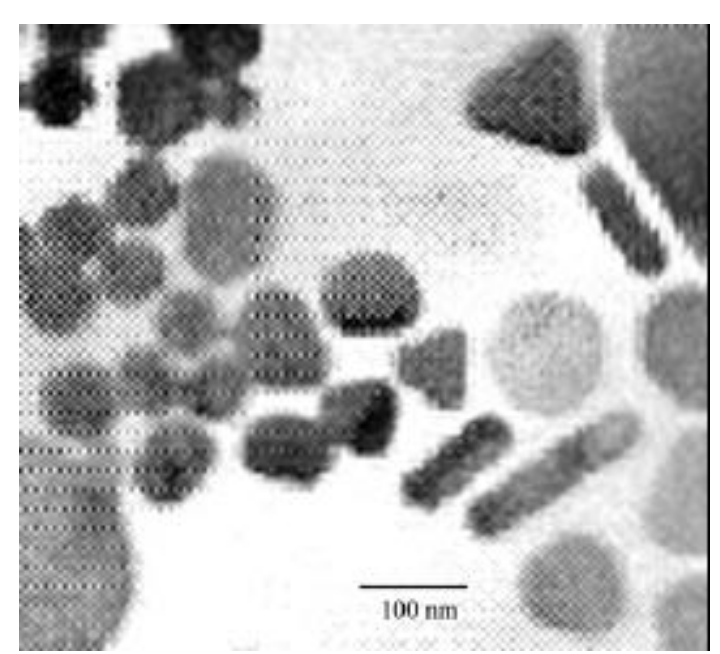

Figure 3. TEM picture for set I. (Condition $\left[\mathrm{HAuCl}_{4}\right]=$ $0.95 \times 10^{-3} \mathrm{~mol} \mathrm{dm}^{-3}$ and $[\mathrm{TX}-100]=0.1 \mathrm{~mol} \mathrm{dm}^{-3}$ ).

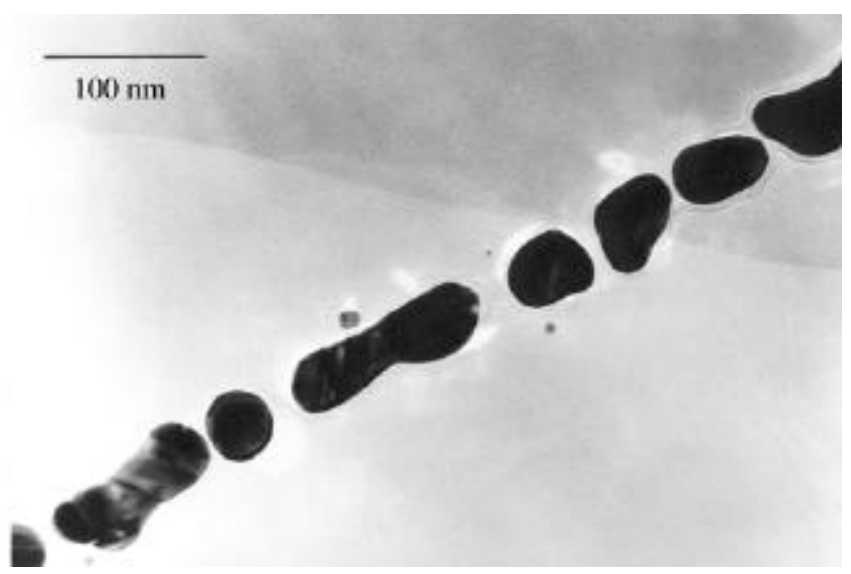

Figure 4. TEM of gold nanoparticles after heating at $60^{\circ} \mathrm{C}$. 
grow haphazardly due to having more diffused $5 d$ orbital in Au-atom. TEM picture of the particles formed in set I is shown in figure 3 .

Lastly, we have studied the heating effect on gold nanoparticles. When $\mathrm{Au}(\mathrm{III})$ solution was irradiated at room temperature $\left(25^{\circ} \mathrm{C}\right)$ with UV-light of high flux density ( 600 lux or more), the pink solution that is formed shows $\lambda_{\max }$ at $523 \mathrm{~nm}$ but if it is irradiated with low flux density (100 lux or less), the resulting blue solution shows $\lambda_{\max }$ at $\sim 533 \mathrm{~nm}$. Then both sets were heated on water bath at $\sim 60^{\circ} \mathrm{C}$ for $30 \mathrm{~min}$. In this temperature range the particles were annealed together and aggregated in linear fashion, which is shown in figure 4. Stronger reducing agent generates nanoparticles with a very narrow size distribution. This is due to the formation of wide number of nucleation centres at a faster rate. Photoactivation under lower flux density (<500 lux) with less number of $\mathrm{Au}(\mathrm{III})$ ions generates a few nucleation centres that easily permits coalescence resulting in larger sized nanoparticles. It is just similar to the seeding growth of crystals.

\section{Conclusions}

This method offers us a simple, easy and reproducible tool to have a control over the size and shape of gold nanoparticles that will undoubtedly help the material scientists in further scientific research.

\section{Acknowledgement}

Authors are thankful to CSIR and DST, New Delhi for financial assistance.

\section{References}

Butte R et al 1999 Philos. Mag. B79 1079

Esumi K, Matsuhisa K and Torigoe K 1995 Langmuir 11 3285

Gachard E, Remita H, Khatouri J, Keita B, Nadjo L and Belloni J 1998 New J. Chem. 1257

Jana N R, Gearhert L and Murphy C J 2001 Chem. Commun. 617

Kreibig U and Vollmer M 1995 Optical properties of metal clusters (Berlin: Springer)

Link S and El-Sayed M A 1999 J. Phys. Chem. B103 8410

Pal A 1998 Talanta 46583

Pal T, Jana N R, Pal A, Creighton J A and Beezer A E $2000 \mathrm{~J}$. Indian Chem. Soc. 7734

Pradhan N, Pal A and Pal T 2001 Langmuir 171800 